\title{
Myosteatosis in NAFLD patients correlates with plasma Cathepsin D
}

https://doi.org/10.1515/bmc-2021-0004

received April 6, 2021; accepted April 23, 2021.

\begin{abstract}
Previously, we have shown that hepatic lipid accumulation induces the secretion of cathepsin D (CTSD), and that plasma CTSD levels are associated with increased inflammation and disease severity in nonalcoholic fatty liver disease (NAFLD). Although it is clear that the liver is a major source of plasma CTSD, it is unknown whether other metabolically active organs such as the muscle, also associate with plasma CTSD levels in NAFLD patients. Therefore, the aim of this study was to explore the relation between lipid accumulation in the muscle (myosteatosis) and plasma CTSD levels in forty-five NAFLD patients. We observed that hepatic steatosis positively associated with plasma CTSD levels, confirming the previously established link between plasma CTSD and the liver. Furthermore, a positive association between myosteatosis and plasma CTSD levels was observed, which was independent of sex, age, BMI, waist circumference and hepatic steatosis. By establishing a positive association between myosteatosis and plasma CTSD levels, our findings suggest that, in
\end{abstract}

*Corresponding author: Ronit Shiri-Sverdlov, Department of Molecular Genetics, NUTRIM School of Nutrition and Translational Research in Metabolism, Maastricht University Medical Center+, Universiteitssingel 50, 6229ER Maastricht, the Netherlands, E-mail: r.sverdlov@maastrichtuniversity.nl

Lingling Ding, Yvonne Oligschlaeger, Inês Magro dos Reis, Tom Houben, Department of Molecular Genetics, NUTRIM School of Nutrition and Translational Research in Metabolism, Maastricht University Medical Center+, Universiteitssingel 50, 6229ER Maastricht, the Netherlands

Toon. J. I. De Munck, Ger. H. Koek, Department of Internal Medicine, NUTRIM School of Nutrition and Translational Research in Metabolism, Maastricht University Medical Center+, Universiteitssingel 50, 6229ER Maastricht, the Netherlands Jef Verbeek, Department of Gastroenterology \& Hepatology, University Hospitals KU Leuven, Herestraat 49, 3000 Leuven Leuven, Belgium

Ger. H. Koek, Department of internal medicine, division of gastroenterology and hepatology, Maastricht University Medical Center. P. Debyelaan 25, 6229 HX Maastricht, the Netherlands addition to the liver, the muscle is also linked to plasma CTSD levels in NAFLD patients. The observed link between myosteatosis and plasma CTSD levels supports the concept of a significant role of the skeletal muscle in metabolic disturbances in metabolic syndrome-related disorders.

Keywords: Plasma CTSD levels; myosteatosis; steatosis; ectopic lipid accumulation; NAFLD.

\section{Introduction}

Due to the growing prevalence of metabolic syndrome (MetS), nonalcoholic fatty liver disease (NAFLD) is an increasing worldwide health problem. Being the most common cause of chronic liver diseases in the Western world [1], NAFLD is a progressive disease ranging from liver steatosis to nonalcoholic steatohepatitis (NASH), hepatic fibrosis, cirrhosis, liver failure and ultimately hepatocellular carcinoma [2, 3]. Given the disease burden associated with NAFLD, more research is needed to elucidate the underlying disease mechanisms as a basis to develop novel therapies.

Previously, our group has shown that, following hepatic steatosis, increased secretion of cathepsin D (CTSD, a lysosomal enzyme) is associated with higher levels of inflammation, disturbed lipid metabolism and disease severity in in vitro and in vivo models for NAFLD, suggesting a pathophysiological role of CTSD in NAFLD [4, 5]. In addition, we observed that plasma CTSD levels are also elevated in NASH patients [6], further emphasizing the association between plasma CTSD levels and NAFLD progression. Of note, studies have shown increased plasma CTSD levels in type 2 diabetes [7], Alzheimer's disease [8] and inflammatory bowel disease [9], suggesting that extra-hepatic organs are also linked to plasma CTSD under pro-inflammatory conditions. Importantly, extrahepatic organs such as the gut, adipose tissue and muscle also induce metabolic disturbances in MetS and NAFLD, 
raising the possibility that next to the liver, also other extra-hepatic organs are linked to plasma CTSD in MetS and NAFLD patients [10-12]. Among the aforementioned extra-hepatic organs, the muscle is increasingly being implicated in the progression of MetS. Accumulating evidence has shown that ectopic fat infiltration in skeletal muscle, referred to as myosteatosis, includes intramuscular and intermuscular lipid accumulation, and is strongly associated with obesity, diabetes and MetS [13, 14]. For instance, intermuscular fat has been shown to be a predictor of insulin sensitivity $[15,16]$, highlighting the link between myosteatosis and systemic metabolism. In addition, intramuscular fat is a metabolically active component of the muscle, which contributes to the secretion of inflammatory cytokines that induce systemic inflammation [14]. Whether the muscle is also linked to plasma CTSD in NAFLD patients has yet to be explored.

Therefore, our study aim was to investigate whether, besides the liver, also the muscle correlates with plasma CTSD levels in NAFLD patients. For this reason, fortyfive NAFLD patients were enrolled in this study, in which muscle signal intensity loss on in-phase and opposedphase imaging (chemical shift MRI) was assessed to determine myosteatosis based on the formula as previously described [17]. Furthermore, fasting blood samples were analyzed for the measurement of glucoseand lipid-related parameters, liver enzymes as well as plasma CTSD levels. Our findings demonstrate a positive association between the degree of myosteatosis and plasma CTSD levels independent of sex, age, BMI, waist and hepatic steatosis in NAFLD patients. Therefore, our data suggest that in addition to the liver, also the muscle is linked to plasma CTSD levels. Taking into account that plasma CTSD aggravates metabolic disturbances, the observed link between myosteatosis and plasma CTSD levels supports the concept of a significant role of the skeletal muscle in metabolic disturbances in metabolic syndrome-related disorders.

\section{Methods and materials}

\section{Subject characteristics}

Forty-five subjects were included in the present study as previously described [17]. All participants had proven NAFLD via liver biopsy or chemical shift magnetic resonance imaging (MRI). Metabolic syndrome was diagnosed based on the International Diabetes Federation (IDF) definition [18]. Exclusion criteria were excessive ethanol consumption (male $>14$ and female >
7 standard beverages per week), causes for secondary hepatic fat accumulation (medication, Wilson's disease, viral infections, starvation or parenteral nutrition and microvesicular steatosis on liver biopsy), pregnancy and breastfeeding, a history of bariatric surgery, liver cirrhosis and/or hepatocellular carcinoma, malignancy(s) within the last 5 years and individuals about to undergo or recovering from a surgical or otherwise medical procedure. All subjects were recruited from Maastricht University Medical Center (MUMC+) and CO-EUR (a second line eating disorder clinic) between September 2015 and October 2018.

Informed consent: Informed consent has been obtained from all individuals included in this study.

Ethical approval: The study was approved by the Medical-Ethical Committee of Maastricht University (ClinicalTrials.gov Identifier: NCT02422238), and was performed in accordance with the principles of the Declaration of Helsinki, as revised in 2008.

\section{Biochemical analyses}

After overnight fasting, venous blood was collected into EDTA tubes that were put on ice after blood collection at Maastricht University Medical Center (MUMC+). After centrifuging (1000 x g; $10 \mathrm{~min} ; 4^{\circ} \mathrm{C}$ ), plasma was snapfrozen in liquid nitrogen and then stored at $-80^{\circ} \mathrm{C}$ until analyses. Aspartate aminotransferase (AST), alanine aminotransferase (ALT), $y$-glutamyl transpeptidase (GGT), bilirubin, Alkaline Phosphatase, total cholesterol, low density lipoprotein cholesterol (LDL-cholesterol), high density lipoprotein cholesterol (HDL-cholesterol), triglyceride, fasting plasma glucose and hemoglobin A1c (HbA1C) were determined using routine analyses at the clinical chemistry department of the Maastricht UMC+ hospital. Plasma CTSD levels were determined using the CTSD enzyme-linked immunosorbent assay according to the manufacturer's protocol (Uscn Life Science, Wuhan, China). The absorbance for CTSD levels was measured on a Benchmark 550 microplate reader (Bio-Rad, Hercules, CA). Skeletal muscle mass index (SMI) (appendicular skeletal mass/height ${ }^{2}$ ) were calculated with dual-energy X-ray absorptiometry (DXA).

\section{Magnetic resonance imaging (MRI) - hepatic fat fraction}

Hepatic fat fraction (hepatic steatosis) was assessed as previously described [17] [19]. In brief, four circular regions of interest (ROI) of $5 \mathrm{~cm}^{2}$ from each MRI section (in total 
3 MRI sections) were drawn in the liver, where artefact, vascular and biliary structures were avoided. Then, the ROI was copied from the in-phase (IP) image to the opposed-phase (OP) image. The mean signal intensity (SI) loss of all 12 ROIs was calculated as previously described $[17,20]$.

\section{Magnetic Resonance Imaging (MRI) - muscle fat fraction}

The MRI technique (chemical shift MRI) was performed to determine muscle fat fraction (myosteatosis) in all participants after a minimal fasting period of 3 hours within one month of the other measurements. MRI images were obtained with a $1.5 \mathrm{~T}$ or 3T Achieva MRI system (Philips, Best, The Netherlands). The muscle fat fraction was measured with the simultaneously obtained 3D fast gradient echo mDixon images as previously described [17]. Parameters employed for 3D fast gradient echo mDixon sequence were for $1.5 \mathrm{~T}$ and $3 \mathrm{~T}$ respectively: flip angle $=15^{\circ}$ and $10^{\circ}$, TR $=5.8$ and $3.4 \mathrm{~ms}$, TE1/TE2 $=2.37 / 4.75$ and $1.19 / 2.37 \mathrm{~ms}$, field-of-view (FOV) $=320 \times 320$ and 384 x $384 \mathrm{~mm}$, acquisition matrix $=288 \times 227$ and $252 \times 209$, slice thickness 5 and $3 \mathrm{~mm}$. The multifidus and erector spinae muscle were segmented bilaterally at the lumbar 1 (L1) level using Weasis software and muscle fat fraction (chemical shift MRI) was calculated with the formula: (SI IP - SI OP)/SI IP * $100 \%$ as previously described [17].

\section{Transient elastography}

Liver stiffness measurements (LSM) were performed in all participants using the FibroScan ${ }^{\circledR}$ (touch 502, Echosens, Paris, France). Both the M probe (3,5 MHz) and the XL probe $(2,5 \mathrm{MHz})$ were available for this study. At least 10 valid measurements, a $60 \%$ success rate and an interquartile range of less than $30 \%$ of the median elasticity were accepted for further analysis. The final result of LSM is the median of the valid (at least 10) individual measurements. The cut-off value for significant fibrosis (histological grade 2 fibrosis (F2)) and advanced fibrosis (F3) were 7.0 $\mathrm{kPa}$ and $8.7 \mathrm{kPa}$, respectively [21]. In the database, we divided fibrosis into no fibrosis $(<7.0 \mathrm{kPa})$ and significant fibrosis $(\geq 7.0 \mathrm{kPa}$ ) groups for further analysis according to the cut-off value of $7.0 \mathrm{kPa}$.

\section{Data statistics}

Statistical analyses were performed using SPSS 25.0 (IBM, Armonk, NY, USA) and Graphpad Prism 6.0 for Microsoft Windows. All data were expressed as means \pm SEM. The differences of myosteatosis between males and females were tested using unpaired t-test in Graphpad Prism 6.0. Pearson's correlations were performed to determine simple correlations between plasma CTSD levels and other parameters. Subsequently, multiple linear regression analyses were performed to analyze the association between plasma CTSD levels and hepatic fat fraction/ fibrosis/myosteatosis, in which plasma CTSD levels were added as dependent variable and either hepatic fat fraction, fibrosis or myosteatosis as independent variables, resulting in model 1 (simple regression), model 2 (model $1+$ adjustment for sex), model 3 (model $2+$ adjustment for age), model 4 (model $3+$ adjustment for BMI) and model 5 (model 4 + adjustment for waist circumference). Additionally, the association between plasma CTSD levels and myosteatosis was also investigated in a multiple linear regression analysis adjusted for sex, age, BMI and waist circumference, using myosteatosis as dependent variable and plasma CTSD levels as independent variable. Only in the multiple linear regression analyses of plasma CTSD levels and myosteatosis, hepatic fat fraction was adjusted in the model 6 (model 5 -hepatic fat fraction). A $p$-value $<0.05$ was considered statistically significant.

\section{Results}

\section{Anthropometric and clinical characteristics of the study participants}

The baseline characteristics of participants are summarized in Table 1. As previously described [17], fortyfive NAFLD patients participated in our study, of which 24 subjects were males and 21 individuals were females, with an average age of $51.1 \pm 1.7$ years old and a mean BMI of $32.9 \pm 0.8 \mathrm{Kg} / \mathrm{m}^{2}$. The average of myosteatosis was 35.57 $\pm 1.65 \%$, ranging from $8.08 \%$ to $54.31 \%$. Finally, plasma CTSD levels ranged from $40150.00 \mathrm{pg} / \mathrm{mL}$ to 1364000.00 $\mathrm{pg} / \mathrm{mL}$, with a mean of $440776.82 \pm 43143.42 \mathrm{pg} / \mathrm{mL}$. 
Table 1: Baseline clinical characteristics of the study participants.

\begin{tabular}{|c|c|c|}
\hline & Mean \pm SEM & Ranges \\
\hline Age, yrs & $51.1 \pm 1.7$ & $20 \sim 65$ \\
\hline $\operatorname{Sex}(M / F)$ & $24 / 21$ & - \\
\hline BMI $\left(\mathrm{kg} / \mathrm{m}^{2}\right)$ & $32.9 \pm 0.8$ & $24.6 \sim 46.3$ \\
\hline Waist circumference $(\mathrm{cm})$ & $108.9 \pm 1.8$ & $85.8 \sim 146.9$ \\
\hline Hip (cm) & $114.0 \pm 1.8$ & $96.0 \sim 154.3$ \\
\hline WHR & $0.96 \pm 0.01$ & $0.73 \sim 1.10$ \\
\hline SBP (mmHg) & $134.5 \pm 2.2$ & $107 \sim 177$ \\
\hline DBP (mmHg) & $81.0 \pm 1.4$ & $62 \sim 115$ \\
\hline Heart rate (bpm) & $66.2 \pm 1.4$ & 49 97 \\
\hline HbA1c (\%) & $5.87 \pm 0.14$ & $4.50 \sim 9.10$ \\
\hline Fasting glucose (mmol/L) & $6.3 \pm 0.3$ & $4.8 \sim 13.0$ \\
\hline Total Cholesterol (mmol/L) & $5.2 \pm 0.1$ & $3.6 \sim 7.7$ \\
\hline Triglycerides (mmol/L) & $2.15 \pm 0.34$ & $0.65 \sim 15.58$ \\
\hline HDL Cholesterol (mmol/L) & $1.26 \pm 0.06$ & $0.50 \sim 2.20$ \\
\hline LDL Cholesterol (mmol/L) & $3.2 \pm 0.1$ & $1.8 \sim 5.4$ \\
\hline GGT (U/L) & $54.9 \pm 7.6$ & $8 \sim 256$ \\
\hline $\operatorname{ALT}(U / L)$ & $46.2 \pm 6.8$ & $7 \sim 272$ \\
\hline AST (U/L) & $32.0 \pm 3.0$ & $11 \sim 118$ \\
\hline Bilirubin (umol/L) & $8.5 \pm 0.6$ & $3.7 \sim 23.1$ \\
\hline Alkaline phosphatase (IU/L) & $95.4 \pm 4.7$ & $52 \sim 186$ \\
\hline Hepatic fat fraction (\%) & $19.51 \pm 1.61$ & $6.62 \sim 45.49$ \\
\hline Fibroscan liver stiffness (Кра) & $6.9 \pm 0.4$ & $3.6 \sim 12.2$ \\
\hline Fibrosis (Yes/No) & $18 / 25$ & - \\
\hline Myosteatosis (\%) & $35.57 \pm 1.65$ & $8.08 \sim 54.31$ \\
\hline Skeletal muscle mass index $\left(\mathrm{kg} / \mathrm{m}^{2}\right)$ & $8.64 \pm 0.19$ & $5.69 \sim 11.20$ \\
\hline CTSD levels (pg/mL) & $440776.82 \pm 43143.42$ & $40150.00 \sim 1364000.00$ \\
\hline
\end{tabular}

Data are mean \pm SEM. BMI, body mass index; WHR, waist/hip ratio; SBP, systolic blood pressure; DBP, diastolic blood pressure; HbA1c, hemoglobin; HDL, high density lipid protein; LDL, low density lipid protein; GGT, gamma-glutamyl transpeptidase; ALT, alanine transaminase; AST, aspartate aminotransferase.

\section{Hepatic steatosis rather than fibrosis is positively associated with plasma CTSD levels}

To validate our previous finding that the liver contributes to plasma CTSD levels, we assessed whether hepatic steatosis and fibrosis associated with plasma CTSD levels in our current NAFLD patients. As shown in Table 2, linear regression analyses were performed by adjusting for sex, age, BMI and waist circumference. Our data showed that hepatic fat fraction positively associated with plasma
CTSD levels (standardized coefficient $\beta=0.300,95 \% \mathrm{Cl}$ : 0.003 0.595, $\mathrm{p}=0.048$ ) in model 1 . Though the association was nearly significant after adjustment for sex (model 2: standardized coefficient $\beta=0.288,95 \% \mathrm{Cl}:-0.012 \sim 0.586$, $\mathrm{p}=0.059$ ), and age (model 3: standardized coefficient $\beta=0.308,95 \% \mathrm{Cl}:-0.002 \sim 0.617, p=0.051$ ), hepatic fat fraction was still significantly associated with plasma CTSD levels after correcting for BMI (model 4: standardized coefficient $\beta=0.316,95 \% \mathrm{Cl}: 0.019 \sim 0.610, \mathrm{p}=0.037$ ) and further adjustment for waist circumference (model 5: standardized coefficient $\beta=0.325,95 \% \mathrm{Cl}: 0.024 \sim 0.623$, 
Table 2: Hepatic fat fraction (steatosis) is positively associated with plasma CTSD levels.

\begin{tabular}{|c|c|c|c|c|c|c|}
\hline \multicolumn{7}{|c|}{ Dependent variable: plasma CTSD levels } \\
\hline \multirow[b]{2}{*}{ Models } & \multicolumn{3}{|c|}{ Hepatic fat fraction } & \multicolumn{3}{|l|}{ Fibrosis } \\
\hline & $\begin{array}{l}\text { Adjusted } \\
\text { R square }\end{array}$ & $\begin{array}{l}\text { Standardized } \\
\text { coefficient } \beta(95 \% \mathrm{Cl})\end{array}$ & $p$ value & $\begin{array}{l}\text { Adjusted } \\
\text { R square }\end{array}$ & $\begin{array}{l}\text { Standardized coefficient } \\
\beta(95 \% \mathrm{Cl})\end{array}$ & $p$ value \\
\hline Model 1 & 0.068 & $\begin{array}{l}0.300 \\
(0.003 \sim 0.595)\end{array}$ & 0.048 & 0.009 & $\begin{array}{l}0.182 \\
(-0.135 \sim 0.504)\end{array}$ & 0.250 \\
\hline $\begin{array}{l}\text { Model } 2 \\
\text { (Model 1+Sex) }\end{array}$ & 0.062 & $\begin{array}{l}0.288 \\
(-0.012 \sim 0.586)\end{array}$ & 0.059 & 0.019 & $\begin{array}{l}0.189 \\
(-0.126 \sim 0.510)\end{array}$ & 0.229 \\
\hline $\begin{array}{l}\text { Model } 3 \\
\text { (Model 2+Age) }\end{array}$ & 0.047 & $\begin{array}{l}0.308 \\
(-0.002 \sim 0.617)\end{array}$ & 0.051 & -0.005 & $\begin{array}{l}0.187 \\
(-0.133 \sim 0.513)\end{array}$ & 0.242 \\
\hline $\begin{array}{l}\text { Model } 4 \\
\text { (Model 3+BMI) }\end{array}$ & 0.134 & $\begin{array}{l}0.316 \\
(0.019 \sim 0.610)\end{array}$ & 0.037 & 0.050 & $\begin{array}{l}0.073 \\
(-0.267 \sim 0.414)\end{array}$ & 0.663 \\
\hline $\begin{array}{l}\text { Model } 5 \\
\text { (Model 4+Waist } \\
\text { circumference) }\end{array}$ & 0.120 & $\begin{array}{l}0.325 \\
(0.024 \sim 0.623)\end{array}$ & 0.035 & 0.026 & $\begin{array}{l}0.094 \\
(-0.274 \sim 0.466)\end{array}$ & 0.603 \\
\hline
\end{tabular}

Data were analyzed by multiple linear regression models: Model 1, simple regression; Model 2, model $1+$ adjustment for sex; Model 3, model 2 + adjustment for age; Model 4, model $3+$ adjustment for BMI; Model 5, model $4+$ adjustment for waist circumference. $p<0.05$ is statistically significant.

(A)

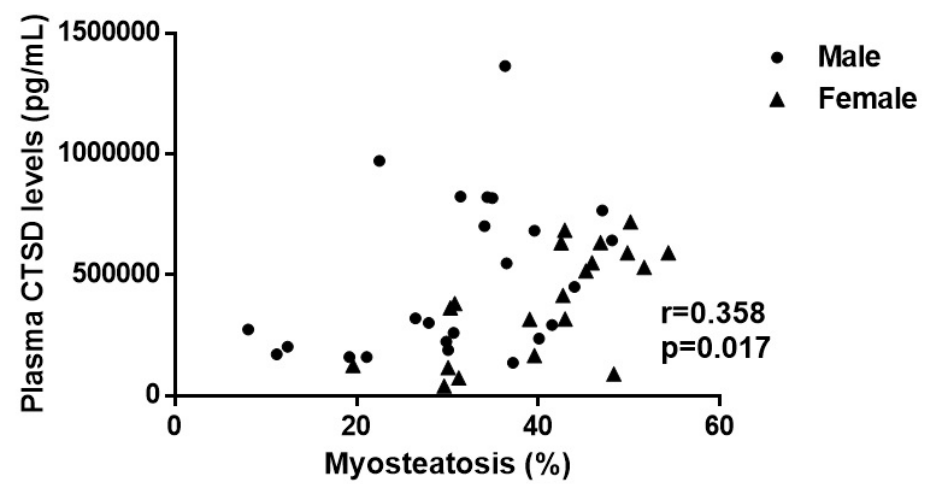

(B)

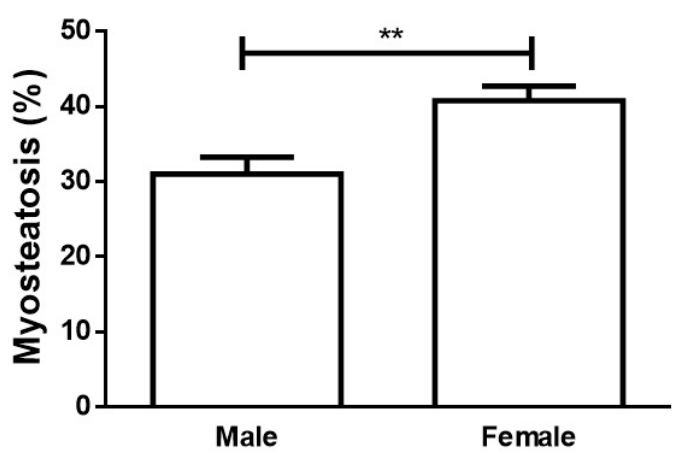

Figure 1: Myosteatosis is gender-dependent and positively correlates with plasma CTSD levels in NAFLD patients. Pearson's correlation was performed to analyse correlation. $p<0.05$ is statistically significant. ${ }^{\star \star} p<0.01$.

$\mathrm{p}=0.035$ ) (Table 2). These data demonstrate that hepatic steatosis is positively associated with plasma CTSD levels independent of sex, age, BMI and waist circumference. As displayed in Supplementary Figure 1, plasma CTSD levels are not significantly different between 'no fibrosis' and 'significant fibrosis' groups. Similarly, we did not observe associations between plasma CTSD levels and fibrosis (as shown in table 2). Altogether, these data demonstrate that hepatic steatosis, but not fibrosis, is independently associated with plasma CTSD levels, confirming the link of the liver to plasma CTSD levels.

\section{The positive association between myosteatosis and plasma CTSD levels independent of sex, age, BMI, waist circumference and hepatic fat fraction}

To investigate whether the muscle also links to plasma CTSD levels, the association between myosteatosis and plasma CTSD levels was analyzed by Pearson's correlation and multiple linear regression analyses. As shown in Figure 1A, we observed that plasma CTSD levels positively 
Table 3: Myosteatosis is positively associated with plasma CTSD levels independent of sex, age, BMI, waist circumference and hepatic fat fraction (steatosis).

\begin{tabular}{llll}
\hline Dependent variable: plasma CTSD levels & & & \\
\hline & Myosteatosis & & p value \\
\hline Models & Adjusted R square & Standardized coefficient $\beta$ (95\% Cl) & 0.017 \\
\hline Model 1 & 0.108 & 0.358 & $(0.067 \sim 0.644)$ \\
Model 2 (Model 1+Sex) & & 0.525 & 0.001 \\
Model 3 (Model 2+Age) & 0.210 & $(0.219 \sim 0.822)$ & 0.001 \\
Model 4 (Model 3+BMI) & 0.194 & 0.537 & 0.007 \\
Model 5 (Model 4+Waist circumference) & & $(0.223 \sim 0.843)$ & 0.471 \\
& 0.177 & $(0.136 \sim 0.799)$ & 0.008 \\
Model 6 (Model 5+Hepatic fat fraction) & 0.280 & 0.474 & $(0.129 \sim 0.812)$ \\
\end{tabular}

Data were analyzed by multiple linear regression models: Model 1, simple regression; Model 2, model $1+$ adjustment for sex; Model 3 , model 2 + adjustment for age; Model 4, model 3 + adjustment for BMI; Model 5, model 4 + adjustment for waist circumference; Model 6 , model $5+$ adjustment for hepatic fat fraction (steatosis). $p<0.05$ is statistically significant.

correlated with myosteatosis $(\mathrm{r}=0.358, \mathrm{p}=0.017)$. Moreover, in Figure 1B, we also found that myosteatosis was genderdependent, showing higher values for myosteatosis in females than males $(\mathrm{p}<0.01)$. To further evaluate whether myosteatosis was dependently or independently associated with plasma CTSD levels, multiple linear regression analysis was performed by adjusting for sex, age, BMI, waist circumference and hepatic steatosis. As displayed in table 3 , myosteatosis positively associated with plasma CTSD levels (model 1: standardized coefficient $\beta=0.358,95 \% \mathrm{Cl}$ : 0.067 0.644, $\mathrm{p}=0.017$ ), even after adjustment for sex (model 2: standardized coefficient $\beta=0.525,95 \% \mathrm{Cl}: 0.219 \sim 0.822, \mathrm{p}=0.001$ ), age (model 3: standardized coefficient $\beta=0.537,95 \% \mathrm{Cl}: 0.223 \sim 0.843$, $\mathrm{p}=0.001$ ), BMI (model 4: standardized coefficient $\beta=0.471$, 95\% Cl: 0.136 0.799, $\mathrm{p}=0.007$ ), waist circumference (model 5: standardized coefficient $\beta=0.474,95 \% \mathrm{Cl}$ : $0.129 \sim 0.812, \mathrm{p}=0.008$ ) and further correcting for hepatic fat fraction (model 6: standardized coefficient $\beta=0.488$, 95\% Cl: 0.165 0.805, $\mathrm{p}=0.004)$. Vice versa, and shown in Supplementary table 1, we also observed that plasma CTSD levels independently associated with myosteatosis independent of sex, age, BMI, waist circumference and hepatic steatosis. As such, these data indicate an independent positive association of myosteatosis with plasma CTSD levels in NAFLD patients, suggesting that, in addition to the liver, also the muscle interacts with plasma CTSD levels.

\section{Discussion}

The exact mechanisms underlying NAFLD development and progression are not yet fully elucidated. Previously, our studies have demonstrated that plasma CTSD levels are associated with the development of hepatic inflammation and dyslipidemia, suggesting that plasma CTSD is a key player in metabolic disturbances in NAFLD $[4,6]$. In the current study, our data shows the positive and independent association between myosteatosis and plasma CTSD levels in NAFLD patients, identifying a link between plasma CTSD levels and the muscle. This observed link supports the concept of a significant role of the skeletal muscle in metabolic disturbances in metabolic syndrome-related disorders.

The consumption of highly caloric, lipid-rich diets is one of the major propellers of MetS and the development of metabolic syndrome associated disorders [22, 23]. Previously, we established lysosomal lipid accumulation as a link between increased cellular uptake of lipids, metabolic disturbances and hepatic inflammation in mouse models for NASH [4]. In addition to interfering 
with proper lysosomal function and thus triggering proinflammatory pathways within cells, lysosomal lipid accumulation causes the leaking of lysosomal contents, culminating in increased secretion of CTSD into the plasma [24] [25, 26]. In line, we have previously shown that NAFLD progression is positively associated with plasma CTSD levels [6], indicating that increased metabolic dysfunction and inflammation are accompanied by increased plasma CTSD levels. Furthermore, our studies show that enhanced plasma CTSD levels in adult NASH patients are likely derived from Kupffer cells [6], thus pinpointing the liver as a source of plasma CTSD following hepatic steatosis and inflammation. In the current study, the observation that myosteatosis in NAFLD patients independently associates with plasma CTSD levels raises the possibility that the muscle, as a metabolic organ, also contributes to plasma CTSD levels. Mechanistically, the increased secretion of CTSD induced by lipid overload in the muscle can be explained by lysosomal exocytosis, a process that is regulated by intracellular $\mathrm{Ca}^{2+}$ channels [25]. Moreover, lipid accumulation in cells is able to induce chronic intracellular $\mathrm{Ca}^{2+}$ overload, subsequently stimulating lysosomes to fuse with the plasma membrane and thus releasing lysosomal enzymes (i.e., CTSD) into the circulation via exocytosis $[27,28]$. Vice versa, and potentially even more interesting, our observed link also raises the possibility that plasma CTSD levels increase myosteatosis, considering plasma CTSD as a mediator inducing ectopic lipid accumulation. Whether myosteatosis leads to plasma CTSD levels, vice versa, or both is a matter of future debate. Nevertheless, our data show a link between myosteatosis and plasma CTSD in NAFLD patients, implying an involvement of the skeletal muscle in MetS-associated disorders.

Previously, myosteatosis has been strongly associated with obesity, diabetes and MetS, suggesting that myosteatosis plays an important role in metabolic disturbances in the context of metabolic syndrome-related disorders [13]. Of note, ample evidence has demonstrated that myosteatosis induces the secretion of local and systemic pro-inflammatory cytokines [14], which are key mediators in the pathogenesis of NAFLD [29]. In addition to pro-inflammatory cytokines, our current study suggests that myosteatosis may further contribute to systemic metabolic dysfunction potentially via increased secretion of CTSD. How plasma CTSD further fuels this metabolic dysfunction is a topic for future research, though interactions with insulin and lipid metabolism appear to be involved players [4, 30].
Furthermore, myosteatosis has been shown to contribute to the aetiology of insulin resistance through the impairment of insulin signalling [31, 32], modulation of adipokine (e.g., adiponectin) secretion [33] and inhibiting nutritive muscle blood flow [34]. Importantly, insulin resistance is recognized as a critical pathophysiological factor in NAFLD [35]. As such, the aforementioned findings suggest that myosteatosis is potentially involved in the pathophysiology of NAFLD by modulating insulin resistance. In line, as previously shown in this cohort, myosteatosis is positively associated with insulin resistance in NAFLD patients [17]. Indeed, our previous studies have demonstrated that inhibiting circulating CTSD reduces plasma insulin levels and improves insulin resistance, suggesting that plasma CTSD aggravates insulin resistance $[5,36]$. As such, these findings provide support for the possibility that myosteatosis contributes to insulin resistance in NAFLD via increased CTSD secretion.

Although the cohort that we used is relatively small, we demonstrate for the first time a positive and independent association between myosteatosis and plasma CTSD levels in NAFLD patients. On one hand, our findings indicate that the muscle, in parallel with the liver, associates with plasma CTSD levels, which may further trigger and exacerbate metabolic dysfunction in NAFLD patients. In addition, whether other metabolically active organs, for instance, adipose tissue also link to plasma CTSD levels in NAFLD should be further addressed in the future and studies on larger cohorts should be conducted to validate our findings. Furthermore, more research is necessary to better understand the underlying mechanism between the interaction of myosteatosis and plasma CTSD levels in the context of MetS.

Acknowledgements: The authors would like to thank all subjects for their participation in the study. This study is funded by the Dutch Organization for Scientific Research (NWO) (Vidi grant no. 016.126.327), TKI-LSH (grant no. 40-41200-98-9306) and VCK (grant no. Swu16.0057-VT). Lingling Ding is supported by the Chinese Scholarship Council with file number 201709110146.

Conflict of interest: Ronit Shiri-Sverdlov is a member of Biomolecular Concept's Editorial Advisory Board.

Data Availability Statement: The datasets generated during and analysed during the current study are available from the corresponding author on reasonable request. 


\section{Reference}

1. Cleveland E, Bandy A, VanWagner LB. Diagnostic challenges of nonalcoholic fatty liver disease/nonalcoholic steatohepatitis. Clin Liver Dis (Hoboken). 2018 Apr;11(4):98-104.

2. Jou J, Choi SS, Diehl AM. Mechanisms of disease progression in nonalcoholic fatty liver disease. Semin Liver Dis. 2008 Nov;28(4):370-9.

3. Younossi Z, Tacke F, Arrese M, Chander Sharma B, Mostafa I, Bugianesi E, et al. Global Perspectives on Nonalcoholic Fatty Liver Disease and Nonalcoholic Steatohepatitis. Hepatology. 2019 Jun;69(6):2672-82.

4. Houben T, Oligschlaeger Y, Hendrikx T, Bitorina AV, Walenbergh SM, van Gorp PJ, et al. Cathepsin D regulates lipid metabolism in murine steatohepatitis. Sci Rep. 2017 Jun;7(1):3494.

5. Khurana P, Yadati T, Goyal S, Dolas A, Houben T, Oligschlaeger Y, et al. Inhibiting Extracellular Cathepsin D Reduces Hepatic Steatosis in SpraguelDawley Rats t. Biomolecules. 2019 May;9(5):E171.

6. Walenbergh SM, Houben T, Rensen SS, Bieghs V, Hendrikx T, van Gorp PJ, et al. Plasma cathepsin D correlates with histological classifications of fatty liver disease in adults and responds to intervention. Sci Rep. 2016 Dec;6(1):38278.

7. Liu L, Chen B, Zhang X, Tan L, Wang DW. Increased Cathepsin D Correlates with Clinical Parameters in Newly Diagnosed Type 2 Diabetes. Dis Markers. 2017;2017:5286408.

8. Chai YL, Chong JR, Weng J, Howlett D, Halsey A, Lee JH, et al. Lysosomal cathepsin $D$ is upregulated in Alzheimer's disease neocortex and may be a marker for neurofibrillary degeneration. Brain Pathol. 2019 Jan;29(1):63-74.

9. Hausmann M, Obermeier F, Schreiter K, Spottl T, Falk W, Schölmerich J, et al. Cathepsin D is up-regulated in inflammatory bowel disease macrophages. Clin Exp Immunol. 2004 Apr;136(1):157-67.

10. Polyzos SA, Kountouras J, Mantzoros CS. Adipose tissue, obesity and non-alcoholic fatty liver disease. Minerva Endocrinol. 2017 Jun;42(2):92-108.

11. Grabherr F, Grander C, Effenberger M, Adolph TE, Tilg H. Gut Dysfunction and Non-alcoholic Fatty Liver Disease. Front Endocrinol (Lausanne). 2019 Sep;10:611.

12. Nachit M, Leclercq IA. Emerging awareness on the importance of skeletal muscle in liver diseases: time to dig deeper into mechanisms! Clin Sci (Lond). 2019 Feb;133(3):465-81.

13. Hausman GJ, Basu U, Du M, Fernyhough-Culver M, Dodson MV. Intermuscular and intramuscular adipose tissues: bad vs. good adipose tissues. Adipocyte. $2014 \mathrm{Dec} ; 3(4): 242-55$.

14. Zamboni M, Gattazzo S, Rossi AP. Myosteatosis: a relevant, yet poorly explored element of sarcopenia. Eur Geriatr Med. 2019 Feb;10(1):5-6.

15. Zoico E, Corzato F, Bambace C, Rossi AP, Micciolo R, Cinti S, et al. Myosteatosis and myofibrosis: relationship with aging, inflammation and insulin resistance. Arch Gerontol Geriatr. 2013 Nov-Dec;57(3):411-6.

16. Sachs S, Zarini S, Kahn DE, Harrison KA, Perreault L, Phang T, et al. Intermuscular adipose tissue directly modulates skeletal muscle insulin sensitivity in humans. Am J Physiol Endocrinol Metab. 2019 May;316(5):E866-79.

17. De Munck TJ, Verhaegh P, Lodewick T, Bakers F, Jonkers D, Masclee AA, et al. Myosteatosis in nonalcoholic fatty liver disease: an exploratory study. Clin Res Hepatol Gastroenterol. 2020 Aug;101500.

18. Alberti KG, Zimmet P, Shaw J. Metabolic syndrome-a new worldwide definition. A Consensus Statement from the International Diabetes Federation. Diabet Med. 2006 May;23(5):469-80.

19. van Werven JR, Marsman HA, Nederveen AJ, Smits NJ, ten Kate FJ, van Gulik TM, et al. Assessment of hepatic steatosis in patients undergoing liver resection: comparison of US, CT, T1-weighted dual-echo MR imaging, and point-resolved 1H MR spectroscopy. Radiology. 2010 Jul;256(1):159-68.

20. Cassidy FH, Yokoo T, Aganovic L, Hanna RF, Bydder M, Middleton MS, et al. Fatty liver disease: MR imaging techniques for the detection and quantification of liver steatosis. Radiographics. 2009 Jan-Feb;29(1):231-60.

21. Wong VW, Vergniol J, Wong GL, Foucher J, Chan HL, Le Bail B, et al. Diagnosis of fibrosis and cirrhosis using liver stiffness measurement in nonalcoholic fatty liver disease. Hepatology. 2010 Feb;51(2):454-62.

22. Lasker S, Rahman MM, Parvez F, Zamila M, Miah P, Nahar K, et al. High-fat diet-induced metabolic syndrome and oxidative stress in obese rats are ameliorated by yogurt supplementation. Sci Rep. 2019 Dec;9(1):20026.

23. Treviño S, Aguilar-Alonso P, Flores Hernandez JA, Brambila E, Guevara J, Flores G, et al. A high calorie diet causes memory loss, metabolic syndrome and oxidative stress into hippocampus and temporal cortex of rats. Synapse. 2015 Sep;69(9):421-33.

24. Hoppe G, O'Neil J, Hoff HF, Sears J. Products of lipid peroxidation induce missorting of the principal lysosomal protease in retinal pigment epithelium. Biochim Biophys Acta. 2004 May;1689(1):33-41.

25. Samie MA, Xu H. Lysosomal exocytosis and lipid storage disorders. J Lipid Res. 2014 Jun;55(6):995-1009.

26. Kornfeld S. Trafficking of lysosomal enzymes in normal and disease states. J Clin Invest. 1986 Jan;77(1):1-6.

27. Kang JK, Kim OH, Hur J, Yu SH, Lamichhane S, Lee JW, et al. Increased intracellular $\mathrm{Ca}+$ concentrations prevent membrane localization of $\mathrm{PH}$ domains through the formation of Ca2+-phosphoinositides. Proc Natl Acad Sci USA. 2017 Nov;114(45):11926-31.

28. Medina DL, Fraldi A, Bouche V, Annunziata F, Mansueto G, Spampanato C, et al. Transcriptional activation of lysosomal exocytosis promotes cellular clearance. Dev Cell. 2011 Sep;21(3):421-30.

29. Stojsavljević S, Gomerčić Palčić M, Virović Jukić L, Smirčić Duvnjak L, Duvnjak M. Adipokines and proinflammatory cytokines, the key mediators in the pathogenesis of nonalcoholic fatty liver disease. World J Gastroenterol. 2014 Dec;20(48):18070-91.

30. Ding L, Goossens GH, Oligschlaeger Y, Houben T, Blaak EE, ShiriSverdlov R. Plasma cathepsin D activity is negatively associated with hepatic insulin sensitivity in overweight and obese humans. Diabetologia. 2020 Feb;63(2):374-84.

31. Petersen KF, Shulman GI. Etiology of insulin resistance. Am J Med. 2006 May;119(5 Suppl 1):S10-6.

32. Timmers S, Schrauwen P, de Vogel J. Muscular diacylglycerol metabolism and insulin resistance. Physiol Behav. 2008 May;94(2):242-51.

33. Vettor R, Milan G, Franzin C, Sanna M, De Coppi P, Rizzuto $R$, et al. The origin of intermuscular adipose tissue and its pathophysiological implications. Am J Physiol Endocrinol Metab. 2009 Nov;297(5):E987-98. 
34. Lee DE, Kehlenbrink S, Lee H, Hawkins M, Yudkin JS. Getting the message across: mechanisms of physiological cross talk by adipose tissue. Am J Physiol Endocrinol Metab. 2009 Jun;296(6):E1210-29.

35. Chen J, Guo Y, Gui Y, Xu D. Physical exercise, gut, gut microbiota, and atherosclerotic cardiovascular diseases. Lipids Health Dis. 2018 Jan;17(1):17.

36. Ding L, Houben $\mathrm{T}$, Oligschlaeger $\mathrm{Y}$, Bitorina AV, Verwer BJ, Tushuizen ME, et al. Plasma Cathepsin D Activity Rather Than Levels Correlates With Metabolic Parameters of Type 2 Diabetes in Male Individuals. Front Endocrinol (Lausanne). 2020 Sep;11:575070. 